\title{
Development of the base of the cochlea: place code shift in the gerbil ${ }^{1}$
}

\author{
David M. Mills *, Edwin W Rubel \\ Virginia Merrill Bloedel Hearing Research Center, Department of Otolaryngology, Head and Neck Surgery, University of Washington, \\ Box 357923, Seattle, WA 98195-7923, USA
}

Received 14 January 1998; revised 21 April 1998; accepted 26 April 1998

\begin{abstract}
Distortion product otoacoustic emission measurements were made at 1/12 octave intervals before and after the injection of furosemide in gerbils aged 15 days after birth to adult, in order to obtain estimates of cochlear amplifier gain as a function of stimulus frequency. The frequency at which the gains went sharply to zero, defined as the 'base cutoff frequency', increased from about $20 \mathrm{kHz}$ to over $50 \mathrm{kHz}$ during development. This increase provides further confirmation of the hypothesis that the place code changes during development in the basal part of the cochlea. If the measured base cutoff frequency is identified with the characteristic frequency at the basal end of the cochlea, as defined by electrophysiological measures, then these emission data can be used to generate a frequency-place map as a function of age. The derived place code shift is consistent with published electrophysiological measures, and can be used to extend these measures. Near the base cutoff frequency, the observed cochlear amplifier gain typically dropped sharply from a relative maximum to zero, over a distance of about a half octave. Specifically, this distance appeared to exhibit a curvilinear variation with age, reaching a maximum of 3/4 of an octave at 19-21 days. After transforming from frequency to place using the map derived from emissions, however, the distance between the extreme base and the place associated with the peak gain decreased monotonically from about $1.2 \mathrm{~mm}$ at age $15-19$ days to $0.6 \mathrm{~mm}$ at maturity. This distance is assumed to be characteristic of the length of the active amplification zone for the cochlear amplifier in the base region. Over the same time period, there was approximately a doubling of the rate of amplification with distance from the base, so that the cochlear amplifier gain at the peak actually changed very little from 15 days to adult. (c) 1998 Published by Elsevier Science B.V. All rights reserved.
\end{abstract}

Key words: Development; Ontogeny; Frequency-place map; Cochlear amplifier; Distortion product otoacoustic emissions; Gerbil

\section{Introduction}

It is well known that the normal mammalian cochlea, when stimulated by a tone, responds with a wave traveling down from the base which peaks at a given location (Johnstone et al., 1986; von Békésy, 1960). The frequency which gives a maximum response at a given place is called the characteristic frequency $(\mathrm{CF})$ associ-

* Corresponding author. Tel.: +1 (206) 616-7540;

Fax: +1 (206) 616-1828; E-mail: dmmills@u.washington.edu

\footnotetext{
${ }^{1}$ Portions of this work were previously presented at the Diversity in Auditory Mechanics conference, Berkeley, CA, June, 1996, and at the 21st Midwinter Meeting of the Association for Research in Otolaryngology, St. Petersburg Beach, FL, February, 1988.
}

ated with that place. In general, the frequency-place relationship is logarithmic, so that for a given cochlea one can define the ratio, $\mathrm{T}$, in $\mathrm{mm} /$ octaves. For example, the relationship was found to be logarithmic for the adult gerbil for $\mathrm{CFs} \geq 2 \mathrm{kHz}$ (Müller, 1996) with a slope of $\mathrm{T}=1.5 \mathrm{~mm} / \mathrm{octave}$. Extrapolating the frequency-place relationship back to zero distance from the base $(\mathrm{z}=0)$ defines the 'base CF', $\mathrm{f}_{0}$. By extrapolation of Müller's published frequency-place relationship, the base $\mathrm{CF}$ for the adult gerbil is $\mathrm{f}_{0} \cong 60 \mathrm{kHz}$. In the present work, distortion product emission measurements are employed to estimate the variation with age of the base CF in the gerbil. By combining these measurements with previous data, we then obtain estimates of the development of the entire frequency-place rela- 
tionship in gerbil, including development of its slope, $\mathrm{T}$ (mm/octave).

The suggestion that the cochlear response changes in important ways during development was made some time ago (Lippe and Rubel, 1983; Rubel, 1978; Rubel et al., 1984; Rubel and Ryals, 1983; reviews: Manley, 1996; Rübsamen, 1992; Sanes and Rubel, 1988; Walsh and Romand, 1992). Specifically, early in development the base of the cochlea appears to respond best to midfrequency tones, but the CFs move to higher frequencies with maturity. This change has important consequences for the development of auditory processing. The place code shift implies that the base $\mathrm{CF}, \mathrm{f}_{0}$, should increase significantly with age during development.

In gerbils, a developmental change in place code has been verified by several methods. First, there were measurements of cochlear potential responses at two locations in the cochlea, the middle of the first and second turns, in gerbils from 12 days old to adult (Arjmand et al., 1988; Harris and Dallos, 1984). The estimated $\mathrm{CFs}$ at the second turn location were between 2 and $3 \mathrm{kHz}$ at all ages, with little or no increase in the mean $\mathrm{CF}$ with age. In contrast, the $\mathrm{CFs}$ in the middle of the first turn increased from about $5-6 \mathrm{kHz}$ at $12-14$ days of age to about $16 \mathrm{kHz}$ at maturity. This increase cannot be due to a change in the physical length of the cochlear duct, which is already of adult size by about 12 days after birth (Harris et al., 1990).

The most direct method of determining the frequency-place map is to measure the CFs of individual neurons and trace their projections back to the cochlea (e.g., Echteler et al., 1989; Liberman, 1982; Manley et al., 1987; Müller, 1996). As noted above, Müller's measurements were quite successful in obtaining a frequency-place map for the adult gerbil cochlea. However, he was able to reconstruct only seven neurons in 18 day old gerbils, and none in younger animals. From the published least square fits to these limited data (Müller, 1996, Fig. 1), the slope of frequency-place relationship at 18 days is estimated to be about $1.8 \mathrm{~mm} /$ octave, compared to $1.5 \mathrm{~mm} /$ octave for the adult. The base $\mathrm{CF}, \mathrm{f}_{0}$, is estimated to be $28 \mathrm{kHz}$ for the 18 day old group, compared to $60 \mathrm{kHz}$ for the adult (Müller, 1996, Fig. 1). The above investigations have been supported by a number of other measurements in developing gerbils (McGuirt et al., 1995; Ryan and Woolf, 1992; Sanes et al., 1989; Schweitzer et al., 1996; Smith and Kraus, 1987; Woolf and Ryan, 1984, 1985, 1988; Woolf et al., 1986; Yancey and Dallos, 1985).

It is important to note that the available data are compatible with the hypothesis that the frequency-place relationship remains approximately logarithmic at earlier ages. However, many additional direct determinations in younger gerbils would be needed to test this hypothesis adequately. In the absence of such measurements, in the present work this hypothesis will be em- ployed for simplicity. Note, however, that the measurements of Arjmand et al. (1988) imply that there are no changes with age at a certain $\mathrm{CF}$, here to be denoted the 'stationary $C F$ ', $\mathrm{f}_{\mathrm{s}}$. Since the base $\mathrm{CF}, \mathrm{f}_{0}$, changes during development, there may be a bend in the log frequency-place relationship at the frequency, $\mathrm{f}_{\mathrm{s}}$, at least at some ages. The available data indicate that this stationary point is $\mathrm{f}_{\mathrm{s}} \sim 1-3 \mathrm{kHz}$ in the gerbil (Arjmand et al., 1988; Müller, 1996).

The difficulties in accurately reconstructing a large number of physiologically defined eighth nerve fibers in developing animals suggests that it would be worthwhile to attempt other, albeit less direct, methods for estimating the $\mathrm{CF}$ and the shape of the cochlear response near the base cutoff frequency as a function of age. Distortion product otoacoustic emissions have proven useful in earlier developmental studies using gerbils from 14 days of age (e.g., Mills et al., 1994; Mills and Rubel, 1996; Norton et al., 1991). Specifically, estimates of the cochlear amplifier gain with age, as a function of frequency, have been obtained from measurements of the changes in otoacoustic emissions with furosemide intoxication (Mills and Rubel, 1996). The gain at the highest frequencies measured (e.g., 32 $\mathrm{kHz}$ ) tended toward zero, when these gain values were extrapolated back to the youngest ages (Mills and $\mathrm{Ru}-$ bel, 1996, Fig. 8). In other words, these data show that the graph of cochlear amplifier gain with frequency at a given age would extrapolate to zero at a given frequency, the 'base cutoff frequency', $\mathrm{f}_{\mathrm{b}}$, and that this base cutoff frequency would be lower in younger animals. At a given age, the cochlear amplifier gain would be expected to be zero for all stimulus frequencies above $\mathrm{f}_{\mathrm{b}}$.

Under circumstances to be described, a connection can be made between the base cutoff frequency, $\mathrm{f}_{\mathrm{b}}$, as defined by the gain of the cochlear amplifier, and the base $\mathrm{CF}, \mathrm{f}_{0}$, defined by the frequency-place map. Consider, for example, the situation where the elements of the cochlear amplifier are functional throughout the cochlear base. For our purposes, the requirement is not that the cochlear amplifier function be mature in all respects, just that its gain be non-zero in most of the base region at the ages tested. Now, consider a pure tone stimulus at a frequency well below the base $\mathrm{CF}$. The stimulus sets up a passive wave traveling down the cochlea from the base. At a certain point, apparently defined by the passive frequency response, active processes begin to feed energy into the traveling wave so that the wave is 'amplified' (Zweig, 1991). This amplification continues over a distance which may be called the length of the active amplification zone (Cody, 1992) and then the energy in the wave rapidly dissipates. It is consistent with the observed behavior to assume that the place where the amplification ceases is also set by the passive frequency response of the basilar membrane. 
This place is typically located at a distance equivalent to about a half octave (in $\mathrm{CF}$ ) from the place where the amplification begins. By definition, the location of the peak response is the 'place' associated with the frequency of stimulation, i.e., the characteristic frequency of that place. Now, consider what must happen as the stimulus frequency increases, to become near to the base CF. As the peak of the response moves toward the base, there is less and less 'room' for the active amplification zone. The decrease in the length of the active amplification zone means that the gain of the cochlear amplifier should decrease as the stimulus frequency becomes near to the base CF. When the stimulus frequency equals the base $\mathrm{CF}$, the gain is zero. Under these circumstances, the base cutoff frequency defined by emissions and the base CF defined by electrophysiological measures are approximately equal: $\mathrm{f}_{\mathrm{b}} \cong \mathrm{f}_{0}$.

A sharp decrease in the gain as the stimulus frequency approaches $f_{0}$ would also be expected from these considerations. The distance over which the sharp decrease occurs will be approximately equal to the length of the active amplification zone of the cochlear amplifier at frequencies near $\mathrm{f}_{\mathrm{b}}$ (Mills and Rubel, 1994, 1996; Mills, 1997, 1998). If this length is denoted b (octaves) and the normal gain of the cochlear amplifier in the base is denoted $\mathrm{G}(\mathrm{dB})$, then there will be a threshold increase with a slope of approximately $G / b$ (dB/octave) due to the cutoff of the active processes as the stimulus frequency approaches the base cutoff frequency. The change will occur over the distance, $b$ (octaves). This threshold increase will be expected to be located at frequencies including the uppermost range of hearing for that animal.

Our earlier data suggest that the elements of the cochlear amplifier are actually functional at near mature levels in the base at the earliest ages (Mills and Rubel, 1996). Under these conditions, in addition to a determination of the base cutoff frequency, a detailed study of the response at the cochlear base would be expected to contribute useful information on the characteristics of the cochlear amplifier at the relevant frequencies. The cochlear base could be considered a naturally occurring system similar to experimentally manipulated systems where outer hair cells (OHC) have been eliminated over a given region. For example, damage of $\mathrm{OHC}$ by impulsive noise led to estimates of the length of the active amplification zone, with a 'substantial part' of the amplification reportedly occurring in a region only $0.5 \mathrm{~mm}$ in length (Cody, 1992). That is, the measured thresholds improved quite rapidly as the stimulus frequency moved below the characteristic frequency associated with the damaged area.

Our previous studies (Mills et al., 1994; Mills and Rubel, 1996) established that there were significant developmental changes in the base cutoff frequency with age, as measured by distortion product emissions. However, measurements of cochlear amplifier gains were obtained at fairly wide frequency intervals, typically $0.5-1$ octave. This precluded an accurate and detailed determination of the characteristics of emissions around the base cutoff frequency, including a determination of the length of the active amplification zone in the basal region and the stability of the emissions just below the base cutoff frequency. Therefore, measurements of an additional 45 gerbils were made with gain determinations at $1 / 12$ octave intervals for frequencies within $1-2$ octaves of the base cutoff frequency. These measurements give a good estimate of the rate of increase of gain as the frequency decreases below the base cutoff frequency.

\section{Methods}

\subsection{Procedures}

The animal preparation and equipment were identical to those previously reported (Mills and Rubel, 1996). Briefly, gerbils from age 15 days after birth to 28 days were anesthetized by subcutaneous injection of a mixture of ketamine hydrochloride (Ketaset; $15 \mathrm{mg} /$ $\mathrm{kg}$ ) and xylazine (Rompun; $5 \mathrm{mg} / \mathrm{kg}$ ). Adult animals ( $>40$ days old) were given the initial anesthesia intraperitoneally, otherwise all procedures were the same. The pinna and outer third of the ear canal were removed, as well as tissue over part of the bulla. A one $\mathrm{mm}$ diameter hole was drilled in the bulla to provide static pressure equalization. The animal was fastened to a custom headholder, and a high frequency acoustic coupler sealed to the ear canal. This custom coupler joined to the ear canal a low noise microphone (ER$10 \mathrm{~B}$, Etymotic), a custom high frequency probe microphone (2530, Larson-Davis) and two inlets for sound delivery (Mills and Rubel, 1996, Fig. 1). The low noise microphone was calibrated in situ by reference to the probe microphone response when a wide band signal was introduced into the ear canal.

Distortion product emission measurements were made in the same way as previously reported (Mills and Rubel, 1996). For this study, the stimulus frequency ratio of the primary tones (frequencies $\mathrm{f}_{1}$ and $\mathrm{f}_{2}$ ) was always $\mathrm{f}_{2} / \mathrm{f}_{1}=1.28$ and the primary amplitudes were equal $\left(\mathrm{L}_{1}=\mathrm{L}_{2}\right)$. The choice of equal level primaries rather than the generally recommended case with $\mathrm{L}_{1}>\mathrm{L}_{2}$ (Mills, 1997) was made for this experiment because of the unique requirements of study near the base cutoff frequency. Near the base cutoff frequency there is a rapid increase in passive threshold with frequency, apparently due to a decrease in the input passive conductivity, i.e., transmission through the middle ear and into the cochlea (Mills and Rubel, 1996, Figs. 9-11). It 


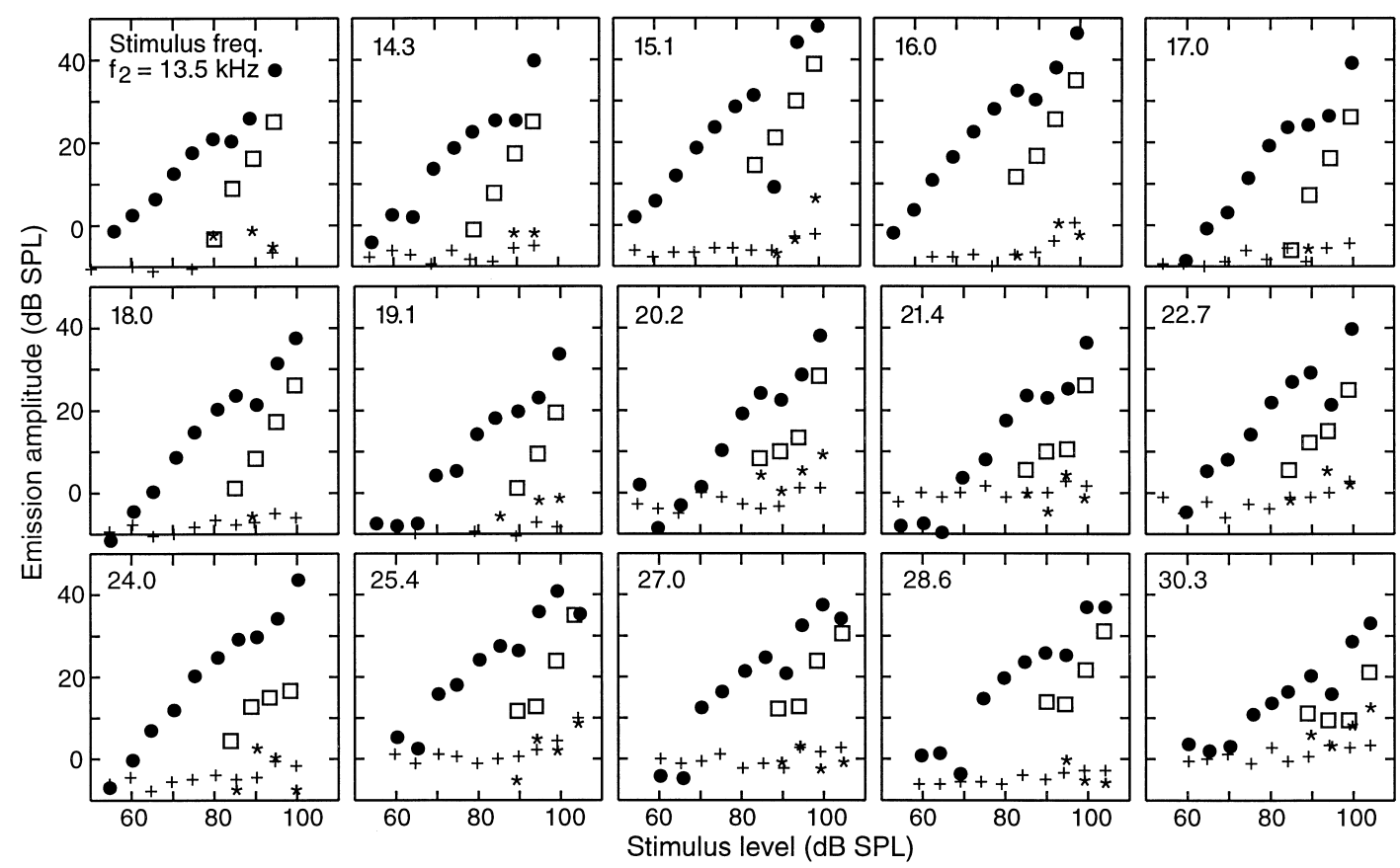

Fig. 1. Representative data set obtained for an individual animal in this study. The subject was a 16 day old gerbil. In each panel the cubic distortion tone (CDT, $2 \mathrm{f}_{1}-\mathrm{f}_{2}$ ) emission amplitude is plotted on the vertical axis as a function of stimulus level on the horizontal. The stimulus always consisted of two, equilevel tones $\left(L_{1}=L_{2}\right)$ with frequency ratio $f_{2} / f_{1}=1.28$. The $f_{2}$ frequency is noted for each panel in the upper left corner. The points for the pre-injection, normal input-output function are indicated by the filled circles, while the squares indicate the points found during the brief 'plateau' phase following furosemide injection (Mills and Rubel, 1994; Mills, 1997). Stars indicate the estimated instrumental distortion, and crosses the measured noise floor.

is likely that a major contributor to this effect is simply the loss of the passive growth of the traveling wave down the cochlea, as the CF moves close to the base. This rapid falloff with frequency means that the intensity of the higher frequency stimulus would have to be larger than usual to compensate for the passive frequency response falloff.

In each preparation, an initial survey of the absolute emission response was first made as a function of frequency, i.e., a 'DP gram' was made. The stimulus frequency, $\mathrm{f}_{2}$, was typically stepped in $1 / 12$ octave intervals from 2 to $64 \mathrm{kHz}$, with a stimulus level of $\mathrm{L}_{1}=\mathrm{L}_{2}=80$ $\mathrm{dB}$ SPL. This allowed an approximate determination of the base cutoff frequency, i.e., the $f_{2}$ frequency where the absolute emission amplitude decreased sharply to the noise floor. Note that, as defined above, the active base cutoff frequency depends on the frequency at which the gain of the cochlear amplifier goes to zero. The gain goes to zero, by definition, when the growth functions before and during furosemide intoxication are approximately the same. One cannot simply use measures of the absolute emission levels, e.g., at lower stimulus levels, to make the determination of the frequency of zero gain. The reason is that either: (1) the amplitude of the emission might still be measurable when the cochlear amplifier gain is zero; or (2) the amplitude of the emission at a given stimulus level might go below the noise floor as the frequency increases while the gain of the cochlear amplifier is still non-zero. Our gain measurements indicate that the second possibility generally does occur.

Following the initial 'DP gram', the frequency range to be investigated for that animal was chosen. This frequency range was typically a 1-2 octave interval with the approximate base cutoff frequency near, but below, the upper limit of this interval. That is, the interval to be studied included the estimated base cutoff frequency. Growth functions were then taken at 1/12 octave steps in frequency, with $5 \mathrm{~dB}$ steps in intensity

Table 1

Age (in days after birth) and numbers of animals in each age group are presented, along with the dosage of furosemide employed for animals in that age group, given as a single intraperitoneal injection

\begin{tabular}{lcc}
\hline Age (days after birth) & Furosemide $(\mathrm{mg} / \mathrm{kg})$ & $N$ \\
\hline 15 & 60 & 4 \\
16 & 80 & 5 \\
17 & 80 & 4 \\
18 & 100 & 4 \\
19 & 100 & 5 \\
21 & 120 & 4 \\
24 & 150 & 4 \\
28 & 200 & 5 \\
$>40$ (Adult) & 300 & 4 \\
Total & & 39 \\
\hline
\end{tabular}

The numbers, $N$, denote the numbers of animals which met all criteria for inclusion in the data analysis. 
up to stimulus levels of $110 \mathrm{~dB}$ SPL. The animal was then given a large intraperitoneal injection of furosemide (see Table 1). Emissions were monitored at several characteristic levels until there was a rapid decline in the responses for low stimulus levels. This rapid decline was typically followed by a relatively unchanging, weak response, the 'plateau' response (Mills and Rubel, 1994; Mills, 1997). Input-output functions were taken during this plateau period, and designated 'post-injection' responses. The same frequencies (in 1/12 octave steps) were employed for the post-injection measurements as for the pre-injection measurements. However, only the highest four or five stimulus intensities were included, since previous studies have shown that the emissions from low level stimuli are well below the noise floor during furosemide intoxication. Following the experiment, the coupler was removed from the gerbil and joined to a long tube of the same approximate diameter as the ear canal. Emission measurements were taken using the same frequencies and levels that were used for the post-injection measurements. This was done to obtain an estimate of the instrumental distortion. It seems preferable to use a completely linear, passive device such as a tube, rather than a post-mortem gerbil ear for these estimates, because of the persistence of emissions from the gerbil ear even an hour or more after death (Schmiedt and Adams, 1981).

The care and use of the animals reported on in this study were approved by the University of Washington Animal Care Committee re: Grant NIH DC 00395, Ontogeny of vertebrate sensory processes.

\subsection{Data analysis}

A data set typical of that acquired for each animal is shown in Fig. 1. Each panel reports emission growth function measurements for the $\mathrm{f}_{2}$ frequency noted in the upper left corner. The horizontal axis in each panel gives the stimulus level in $\mathrm{dB}$ SPL, and the vertical axis gives the emission amplitude at $2 \mathrm{f}_{1}-\mathrm{f}_{2}$, in $\mathrm{dB}$ SPL. All panels have the same axes. In every panel the normal, pre-injection, growth function measurements are shown by filled circles, and the post-injection measurements by open squares. The instrumental distortion estimates are indicated by the stars, and the noise floor estimates by the small crosses.

At all $\mathrm{f}_{2}$ frequencies, there was a clear difference between the normal, pre-injection emission growth functions and those measured with acute furosemide intoxication. At the mid frequencies, note panels for $\mathrm{f}_{2}=16-19 \mathrm{kHz}$ in particular, the post-injection emissions closely followed a power law, i.e., the growth function followed a straight line on a dB-dB plot. In this case, the slopes were about 1.5. This is shown in more detail in Fig. 2, for the $18 \mathrm{kHz}$ panel. Note that the pre-injection emissions at low stimulus levels were also approx- imately straight, with a slope approximately equal to the post-injection growth functions. It was earlier suggested (Mills and Rubel, 1996) that the horizontal shift between the pre- and post-injection growth functions, denoted $G_{c}$ in Fig. 2, was related to the cochlear amplifier gain. Model calculations have confirmed this general relationship, and suggested that the shift measured by emissions typically underestimates the true gain by about 10 dB (Mills, 1997). However, these model calculations result in a growth function slope of $3: 1$. As noted, the measured slopes were closer to 1.5 in the present study, where $\mathrm{L}_{1}=\mathrm{L}_{2}$, and were about 2.0 in a previous study, with $\mathrm{L}_{1} / \mathrm{L}_{2}=10 \mathrm{~dB}$ (Mills and Rubel, 1996). The reasons that the observed slopes are generally lower than the theoretical slope are not known, so that the correct numerical relationship between the actual gain and that estimated by emissions remains uncertain. The general reason for the difference may be that there is a weak mutual suppression operating in the cochlea even at low stimulus levels, contrary to the assumption of the simplest model calculations (Mills, 1997).

In any case, the horizontal shift between pre- and post-injection growth functions, as seen in Fig. 2, is certainly proportional to the gain, and in this study it is the relative gain as a function of frequency which is of importance, not the absolute gain itself. Therefore, the difference between the pre- and post-furosemide growth functions, denoted $G_{c}$, will be used without correction as an estimate of the relative cochlear amplifier gain (Fig. 2). However, it must be emphasized that the difference, $\mathrm{G}_{\mathrm{c}}$, is a measure of the cochlear amplifier gain, and is not necessarily equal to the cochlear amplifier gain (Mills, 1997). Comparison of the values obtained for this measure to the gains estimated from other methods such as emission suppression and eighth nerve tuning curves in gerbils will be deferred to the discussion.

At lower frequencies with this animal (Fig. 1), it was not obvious that the low stimulus level pre-injection growth functions became parallel to the post-injection functions. Measurements would be required to go to very low stimulus levels to adequately test this. Whenever measurements have been extended to such stimulus levels, the normal growth functions were found to steepen eventually, so that they became parallel with the post-injection functions (Mills and Rubel, 1994). In this regard, model calculations also indicate that more accurate results for gain estimates are generally obtained using growth functions with $\mathrm{L}_{1}>\mathrm{L}_{2}$ (Mills, 1997, 1998). As noted above, however, for this study equilevel stimuli were used because of the hypothesis that this would increase the accuracy of measurements near the base cutoff frequency. Therefore, measurements at lower frequencies are not expected to be as accurate as in previous reports (Mills and Rubel, 
1996), and the data analysis and interpretation in this report will be focused on measurements near the base cutoff frequency.

At all $\mathrm{f}_{2}$ frequencies, the difference between the preand post-injection functions in Fig. 1 clearly reflects a significant contribution from the cochlear amplifier. The difference was approximately constant until the highest frequencies. At the same time, there was a gradual shift to the right for both curves as frequency increased. That is, the gain was approximately constant while the threshold increased steadily with increasing frequency. At the highest frequencies, however, the gain dropped abruptly. As one might expect, at the same time the emission amplitude decreased sharply. The difficulty of measurement at these frequencies was further compounded by a general increase in the microphone noise floor with frequency (Fig. 1). The result was that, precisely at the point at which one would like to measure most carefully, there were frequently insufficient points above the noise floor to reliably determine the slopes of the pre- and post-injection growth functions. This is illustrated by the panel for $\mathrm{f}_{2}=30.3$

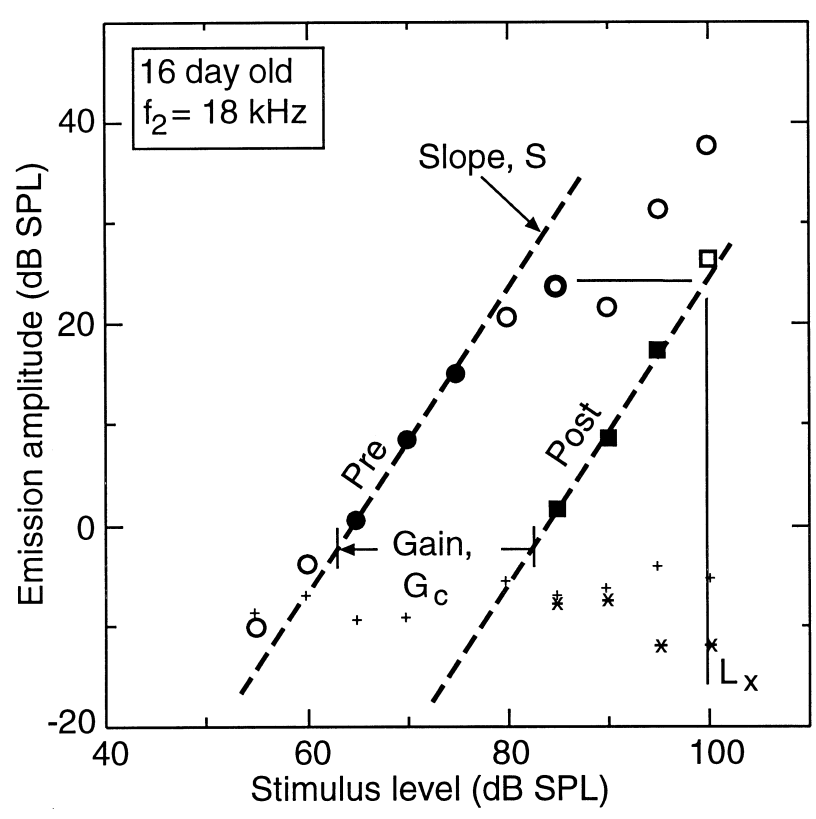

Fig. 2. The growth function for $\mathrm{f}_{2}=18 \mathrm{kHz}$ for the same animal shown in Fig. 1. Same conventions as Fig. 1, except that only points used for the least square fits are shown filled in. Criteria were (1) all points were omitted which were $5 \mathrm{~dB}$ or less above the noise floor or instrumental distortion estimates at that stimulus level, (2) only the lowest three remaining points were used for a least squares fit, and (3) pre- and post-injection lines were required to be parallel. The lines indicate the best fit lines with the slope $S$ (here, $\mathrm{S}=1.5$ ). The distance between these two lines gives the estimate of the gain, $\mathrm{G}_{\mathrm{c}}$. Here, $\mathrm{G}_{\mathrm{c}}=19 \mathrm{~dB}$. The intercept between the relative maximum found before the notch with the post-injection line determines the 'active-passive crossover level', denoted $\mathrm{L}_{\mathrm{x}}$. Here, $\mathrm{L}_{\mathrm{x}}=100$ dB SPL. 'Pre' and 'post' indicate measurements before furosemide injection and during the post-injection plateau.

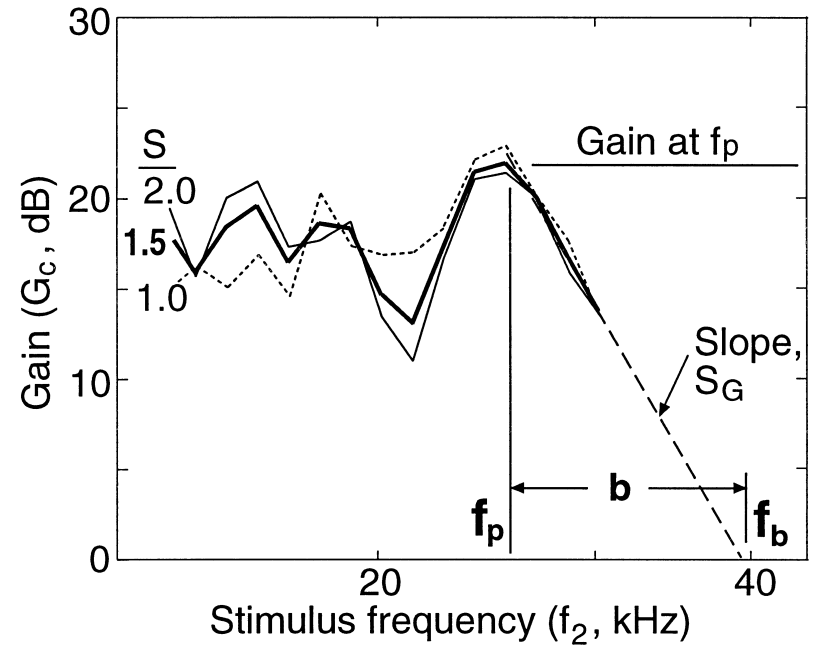

Fig. 3. Estimated gain as a function of frequency for the same 16 day old gerbil shown in Figs. 1 and 2. The gain was estimated for three different slopes, S. Definitions to be employed later are made using the curve $\mathrm{S}=1.5$ as illustrated. There was a peak in gain which could be identified, above which the gain tended rapidly toward zero. The frequency at the peak gain was denoted $f_{p}$. A linear, least squares fit to the data points for frequencies above and including the peak gain determined the slope $S_{G}$, as shown. The intercept of this line with zero gain gives the 'base cutoff frequency', $\mathrm{f}_{\mathrm{b}}$. The distance between $\mathrm{f}_{\mathrm{p}}$ and $\mathrm{f}_{\mathrm{b}}$ is $\mathrm{b}$ (octaves) $=\log _{2}\left(\mathrm{f}_{\mathrm{b}} / \mathrm{f}_{\mathrm{p}}\right.$ ).

$\mathrm{kHz}$ in Fig. 1. For this animal, emission amplitudes at $32 \mathrm{kHz}$ and above were generally too low to measure.

For all of the above reasons, it was decided to adopt a rule based system of estimating the horizontal shift between the pre- and post-furosemide growth functions. The intent was to establish a quantitative method to estimate the effective gain of the cochlear amplifier without the derived gain values being overly sensitive to the assumed or measured slopes of the growth functions, and to avoid possible bias in the estimates. First, all points less than $5 \mathrm{~dB}$ above the measured noise floor or instrumental distortion levels were removed. To increase accuracy, this cutoff was increased to $10 \mathrm{~dB}$ for the pre-injection growth functions when there were judged to be sufficient points at low stimulus levels, i.e., where the sensitivity of the system was high. Then, the three remaining points at the lowest stimulus levels were chosen for each growth function, to define $\mathrm{G}_{\mathrm{c}}$. These points are indicated by the filled in symbols in the example in Fig. 2. In a few cases, after removing points too close to noise or instrumental distortion, there were less than three remaining points in the post-injection data, and in these cases all of the remaining points were used to determine the location of the best fit line. If there were no points left, of course, the data for that frequency had to be discarded.

In general, in order to properly estimate the gain from the experimental data, it is necessary to assume that the two fitted lines are parallel, as shown in Fig. 2. 


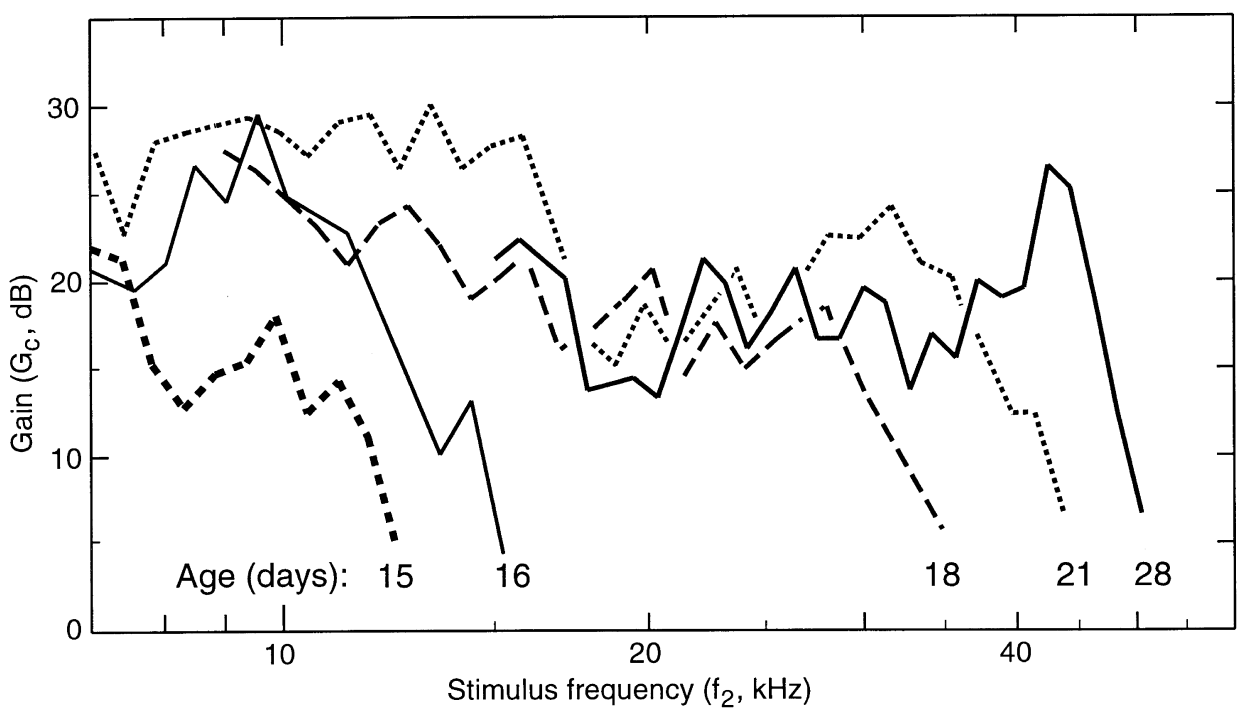

Fig. 4. Representative examples of gain vs. frequency curves for five individual gerbils of different ages, from 15 to 28 days after birth.

The least square fits to the data points for each line can then be found, the difference being the gain, $\mathrm{G}_{\mathrm{c}}$. With the assumptions made for the rule-based derivation, i.e., using only the three lowest data points above the noise for each line, it is apparent that the derived gain values will be relatively insensitive to the slope of these parallel lines. This is demonstrated in Fig. 3, which shows the calculated gain values, $G_{c}$, as a function of the slope of the parallel lines, S, varied from 1.0 to 2.0. The derived gain values as a function of frequency do not systematically vary as a function of the slopes picked for these calculations. Further, in the most important region, that near the base cutoff frequency, all three slopes give essentially the same curves.

Because the estimated gains between the two lines are insensitive to the actual slope assumed, it was therefore decided to use a slope $\mathrm{S}=1.5$ in the estimates that follow. This approach provides one of many objective ways to estimate the variation of gain as a function of frequency for each animal. We have tried other methods, such as visually estimating the best slope to be used for each animal. The results are essentially the same as those reported below.

In addition to the gain measure, $\mathrm{G}_{\mathrm{c}}$, the other important quantity obtained was the crossover stimulus level, $\mathrm{L}_{\mathrm{x}}$. This is the stimulus level required to complete the transition between the pre- and post-furosemide response (Fig. 2). As shown, $L_{x}$ is defined by the intersection of the horizontal line denoting the relative maximum in the pre-injection growth function with the best fit line to the post-injection growth function. This crossover level provides a natural 'threshold' measure for what we define as the 'passive' response. That is, $\mathrm{L}_{\mathrm{x}}$ represents the stimulus level required to reach a response characteristic of the passive response with the criterion level set by cochlear mechanics. This threshold measure therefore gives an estimate of the relative conductivity of the entire passive input transmission path (Mills et al., 1994; Mills and Rubel, 1996; Mills, 1997).

Fig. 3 also provides definitions of terms which were used to characterize the gain behavior near the base cutoff frequency. As shown, the gain typically rose rapidly as the frequency decreased below the base cutoff frequency, to reach a relative maximum in the gain estimate, $\mathrm{G}_{\mathrm{c}}$, at the 'peak' frequency, $\mathrm{f}_{\mathrm{p}}$. The line with the slope, $S_{G}$, represents the linear, least squares fit for the points including and above the gain maximum (here, for $\mathrm{S}=1.5$ ). Only animals with three or more points determining this slope were admitted into the final data analysis. Of the 45 animals initially measured, six had to be excluded by this criterion. About half of the 39 animals remaining had either three or four data points at and above the frequency of peak gain, the rest had five to seven points which were used to determine the least squares fit for $\mathrm{S}_{\mathrm{G}}$. The example shown in Fig. 3 is one of those with four points at and above the gain maximum. The extrapolation of the fitted line of slope $\mathrm{S}_{\mathrm{G}}$ to zero gain determined the base cutoff frequency, $\mathrm{f}_{\mathrm{b}}$. The distance, $\mathrm{b}$, over which the gain rose sharply was also defined as shown. This distance, $b$, is measured in octaves. If the slope, $\mathrm{T}$ ( $\mathrm{mm} /$ octaves), for the frequency-place relationship is known, b can be converted to an actual distance.

There were relatively large variations in estimated gain at stimulus frequencies below the peak gain frequency, $f_{p}$ (shown in Figs. 3 and 4). Comparison with earlier results (Mills and Rubel, 1996) suggests that the variations in these estimates is at least partly be due to the fact that equal level primaries are not optimal for gain estimates in general, although these may be appro- 
priate for measurements near the base cutoff frequency as discussed above. Therefore, only the gain estimates at frequencies from $f_{p}$ to $f_{b}$ are used for quantitative data analysis in this report.

\section{Results}

\subsection{Responses as a function of stimulus frequency}

Representative examples of the variation of gain, $G_{c}$, with stimulus frequency $\mathrm{f}_{2}$ are shown in Fig. 4, for several ages between 15 and 28 days old. In all cases there was a relative maximum, followed by a sharp decline of the gain as frequency increased. The frequency at which this sharp decline occurred increased rapidly as the animals aged.

As discussed in Section 2, the high frequency end of the individual gain vs frequency curves were analyzed according to the 'model' response shown in Fig. 3. That is, the frequency at which the relative peak gain occurred was estimated, and a linear decrease above this frequency assumed, with a least squares fit to determine the mean slope. The resulting means (and standard errors of the mean) as a function of frequency are presented in Figs. 5 and 6. Fig. 5B presents the variation with age of the mean gain measure at the peak frequency, $\mathrm{f}_{\mathrm{p}}$. At all ages, the gain at the peak frequency was about 20-25 $\mathrm{dB}$ and there were no significant changes as a function of age. This is quite remarkable because the frequency associated with this gain, $\mathrm{f}_{\mathrm{p}}$, increased steadily with age, as is shown below. Further, earlier work indicated that the gain at a given, mid range frequency increased rapidly from 15-20 days, but then subsequently decreased with maturity (Mills and Rubel, 1996). It was suggested that the increase was driven by the rapid maturation of the endocochlear potential (EP) during the 15-20 day period. For comparison, Fig. 5A presents the variation with age of the $\mathrm{EP}$, and of the gain, $\mathrm{G}_{\mathrm{c}}$, at $8 \mathrm{kHz}$ (from Mills and Rubel, 1996). Clearly the gain at the 'leading edge' of the gain vs frequency response, i.e., the gain at the frequency $f_{p}$ (defined in Fig. 3) does not show the strong variation with age that the gain at $8 \mathrm{kHz}$ does.

The lower panel in Fig. 5C presents the variation with age of the crossover stimulus level, $\mathrm{L}_{\mathrm{x}}$, measured at the frequency associated with the peak gain. Note that, while this is also an estimate taken at a steadily increasing frequency $\left(f_{p}\right)$, there was clearly an improvement with age. This is a particularly important measure because it is a measure of a threshold at a fixed location, the extreme base of the cochlea. This threshold is therefore not affected by passive growth of the traveling wave down the basilar membrane, but is expected to be primarily set by middle ear conductivity. The data in Fig. 5C suggest that middle ear transmission must
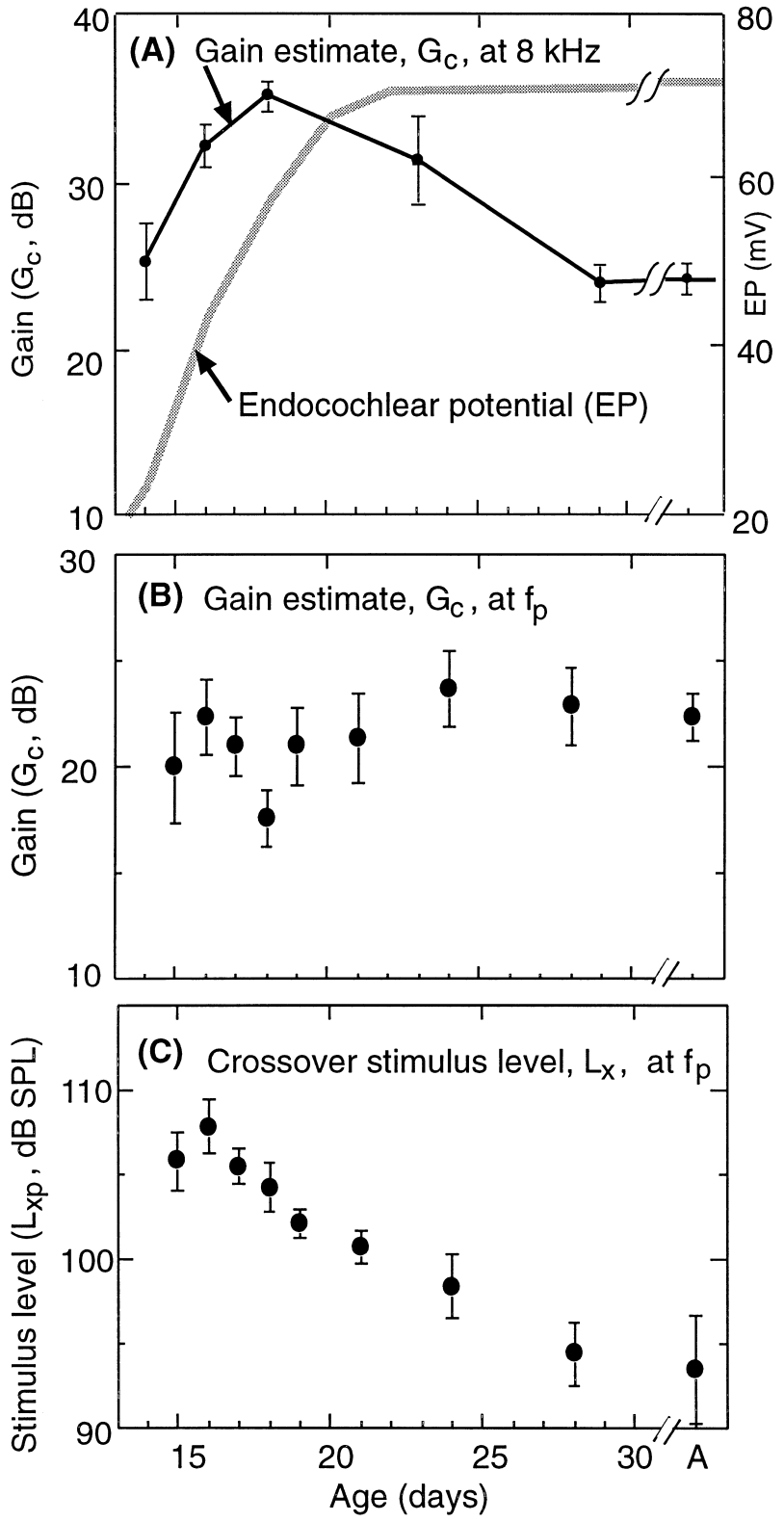

Fig. 5. Mean variation of two quantities associated with the base cutoff frequency change during development, as defined in Fig. 3. In all cases, error bars indicate standard error of the mean. For comparison, the top panel (A) shows the gain response, $G_{c}$, measured at a fixed frequency $\left(\mathrm{f}_{2}=8 \mathrm{kHz}\right.$ ) from previous work (Mills and $\mathrm{Ru}-$ bel, 1996), and the variation of endocochlear potential (EP) with age as measured by Woolf et al. (1986). Panel B shows the gain estimate for the gain at the peak frequency, $f_{p}$ (Fig. 3). Panel $C$ shows the mean stimulus level required at a frequency equal to $f_{p}$ to reach the active-passive transition as defined in Fig. 2. The letter A on the horizontal axis indicates mean values for adult animals ( $>40$ days old).

improve with age, with a threshold improvement of about $15 \mathrm{~dB}$ from 16 to 28 days, as measured at the frequency $f_{p}$, even as, at the same time, this frequency increases from 18 to $40 \mathrm{kHz}$ over the ages examined. An improvement of this order is compatible with exist- 

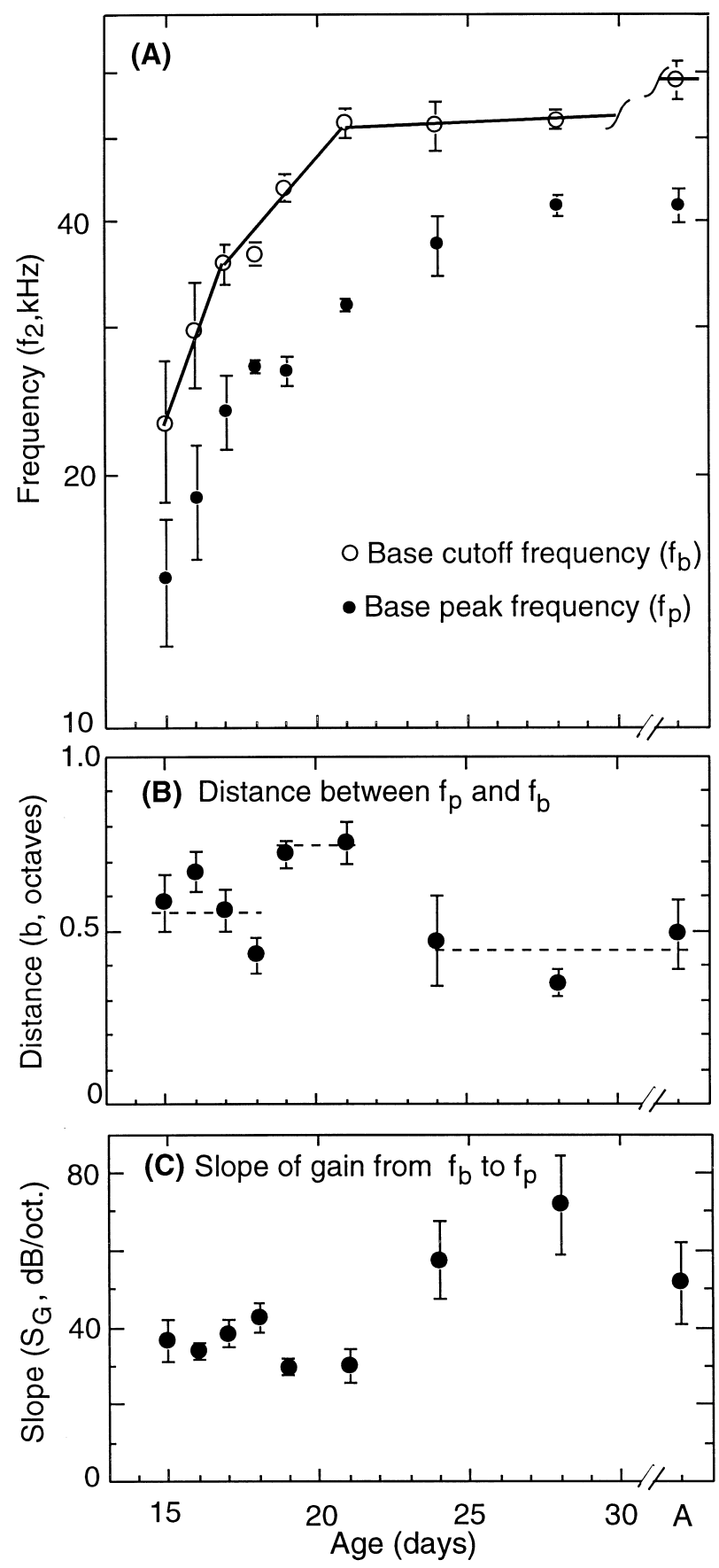

Fig. 6. Mean variation with age of remaining quantities defined in Fig. 3. A: Variation of the frequency of the peak gain, $f_{p}$, and base cutoff frequency, $f_{b}$, with age. The solid line indicates the values which were used to determine the frequency-place transform in Fig. 7. $B$ : The distance, $b$, between the peak frequency, $f_{p}$, and the frequency at which the gain is extrapolated to zero, $\mathrm{f}_{\mathrm{b}}$. The horizontal dashed lines indicates the mean value during the relevant age ranges. $\mathrm{C}$ : The slope of the gain above the peak, $\mathrm{S}_{\mathrm{G}}$, in $\mathrm{dB} /$ octave.

ing measurements of middle ear function in gerbil. At $32 \mathrm{kHz}$, for example, there is about $30 \mathrm{~dB}$ total threshold improvement from 16 days to adult. Because there is only about $12 \mathrm{~dB}$ improvement over the same period when the stapes is driven directly, this suggests that 18
$\mathrm{dB}$ of the total improvement occurs in middle ear transmission (compare Fig. 1, Woolf and Ryan, 1984, to Fig. 3, Woolf and Ryan, 1988; also see Mills and Rubel, 1998).

The variation of the base cutoff frequency, $\mathrm{f}_{\mathrm{b}}$, and of the peak frequency, $f_{p}$, are shown in Fig. 6A. The distance between these frequencies, $\mathrm{b}$, is given in Fig. 6B and the slope of the gain between them is given by Fig. 6C. It is clear that the base cutoff frequency increased sharply with age from 15 to 21 days, with a total change more than an octave. The peak gain frequency generally kept pace, except when it appeared to lag during the period 19-21 days.

Fig. 6B shows the resulting distance in octaves between these two frequencies, $b=\log _{2}\left(f_{b} / f_{p}\right)$. Analysis of variance yielded a significant $F$ value as a function of age $\left(F_{9,39}=4.0 ; P<0.002\right)$. Examination of the data suggests that there was no significant change in this distance during the first period, from 15 through 18 days. The mean value during this period is indicated by the horizontal dashed line, and was about 0.6 octave There then appeared to be an increase in the distance, b, at 19-21 days, to a value of about 3/4 octave. With maturity, the distance decreased sharply to about 0.4 octave.

In contrast, the slope of the 'leading edge', $\mathrm{S}_{\mathrm{G}}$, was approximately constant from 15 to 21 days, equal to about $35 \mathrm{~dB} /$ octave. It then increased to about $60 \mathrm{~dB} /$ octave from 24 days on (Fig. 6C). The apparent increase in variance in the older age groups is difficult to assess because of decreases in the signal to noise ratio of the microphone at the high frequencies characteristic of the base cutoff frequency at the older ages, as discussed in Section 2.

\subsection{Conversion from frequency to place}

Two of the quantities presented above, the distance between the frequency of the peak response and the base cutoff frequency, $b$, and the slope of this decrease, $\mathrm{S}_{\mathrm{G}}$ to this point have been expressed in units of octaves (Fig. 6B,C). If the ratio, $\mathrm{T}$ (mm/octave), is known for the relevant frequency range and age, these quantities can be converted to distance along the cochlear duct. As noted above, a direct measure of the characteristic frequency vs distance gives $\mathrm{T}=1.5 \mathrm{~mm} /$ octave for the adult gerbil (Müller, 1996) for frequencies above $2 \mathrm{kHz}$. However, the transformation ratio is poorly known for younger animals: direct measurements suggest a ratio of about $1.8 \mathrm{~mm} /$ octave at 18 days of age for frequencies above $1-2 \mathrm{kHz}$ (Müller, 1996), but this estimate is based on few data. The emission data in this report provide an internally consistent, alternative method of obtaining the variation of the transformation ratio with age, using the following assumptions: (1) the adult ratio is $1.5 \mathrm{~mm} /$ octave; (2) the ratio does not change signifi- 
cantly with age at a certain place, i.e., for CFs at the 'stationary' $\mathrm{CF}, \mathrm{f}_{\mathrm{s}}$; (3) at any age, the base cutoff frequency obtained by extrapolating the cochlear amplifier gain to zero (i.e., $\mathrm{f}_{\mathrm{b}}$ ) is approximately equal to the $\mathrm{CF}$ of the extreme base of the cochlea, i.e., equal to $\mathrm{f}_{0}$, so that $\mathrm{f}_{\mathrm{b}} \cong \mathrm{f}_{0}$.

From measurements in the second turn of the cochlea, Arjmand et al. (1988) suggested that the characteristic frequency at this location, about $2.6 \mathrm{kHz}$, did not change much with age. However, the limited data of Müller (1996) suggest that the 'stationary' CF might be less than $1 \mathrm{kHz}$. The value for the stationary frequency $\mathrm{f}_{\mathrm{s}}=2 \mathrm{kHz}$ will be taken for illustration in the present work. The transformation ratio, $\mathrm{T}$ ( $\mathrm{mm} / \mathrm{octave}$ ), can be then be calculated from $\mathrm{T}=\mathrm{L}_{\mathrm{sb}} /\left(\log _{2}\left(\mathrm{f}_{\mathrm{b}} / \mathrm{f}_{\mathrm{s}}\right)\right)$ where $L_{s b}$ is the distance $(\mathrm{mm})$ from the stationary point to the base of the cochlea $(z=0)$. For the gerbil cochlea from 12 days on, $\mathrm{L}_{\mathrm{sb}} \cong 7.3 \mathrm{~mm}$ for $\mathrm{f}_{\mathrm{s}}=2 \mathrm{kHz}$, assuming a total length of $11.1 \mathrm{~mm}$ (Müller, 1996). It is important to note that the estimated slopes are only weakly dependent on the exact value of the assumed stationary frequency, $\mathrm{f}_{\mathrm{s}}$.

Using the values for $\mathrm{f}_{\mathrm{b}}$ in Fig. $6 \mathrm{~A}$ indicated by the solid line, the transformation ratio obtained is presented in Fig. 7A. The heavy solid line indicates the mean transformation ratio itself and the light lines the uncertainty in $\mathrm{T}$ (standard error of the mean) due to the observed variation in the base cutoff frequency at a given age. Using this transformation ratio, the transformed slope $\mathrm{S}_{\mathrm{G}^{\prime}}{ }^{\prime}(\mathrm{dB} / \mathrm{mm})$ and the distance, $\mathrm{b}^{\prime}(\mathrm{mm})$ are found as shown in Fig. 7B,C. The error bars represent the uncertainties in the mean values due to the combination of variance in the measured values and in the transformation ratio. The variation with age of both the transformation ratio and the frequency-place map found using these assumptions will be compared with the existing data from other studies below.

While there was still considerable variability in the estimates of the distance, $b^{\prime}$, the response was no longer significantly curvilinear when expressed in distance units. Rather, it appears that the distance, $b^{\prime}$, over which the cochlear amplifier response rose to a maximum was about $1.2 \mathrm{~mm}$ during 15-21 days of age, then decreased to about half this length, $0.6 \mathrm{~mm}$, by maturity. The slope associated with this rise, $\mathrm{S}_{\mathrm{G}}{ }^{\prime}$, was about $20 \mathrm{~dB} / \mathrm{mm}$ for the younger ages, then doubled, increasing to about $40 \mathrm{~dB} / \mathrm{mm}$ with maturity.

The distance, $b^{\prime}$, over which the cochlear amplifier gain falls to zero from the relative maximum is expected to be approximately equal to the length of the active amplification zone, at the frequency $\mathrm{f}_{\mathrm{p}}$ (Mills and $\mathrm{Ru}$ bel, 1994, 1996; Mills, 1997). The interpretation of the present results is then that the length of the active amplification zone was about $1.2 \mathrm{~mm}$ in the young gerbil, near the onset of hearing. By maturity, however, this length had decreased to $0.6 \mathrm{~mm}$. Since the total gain
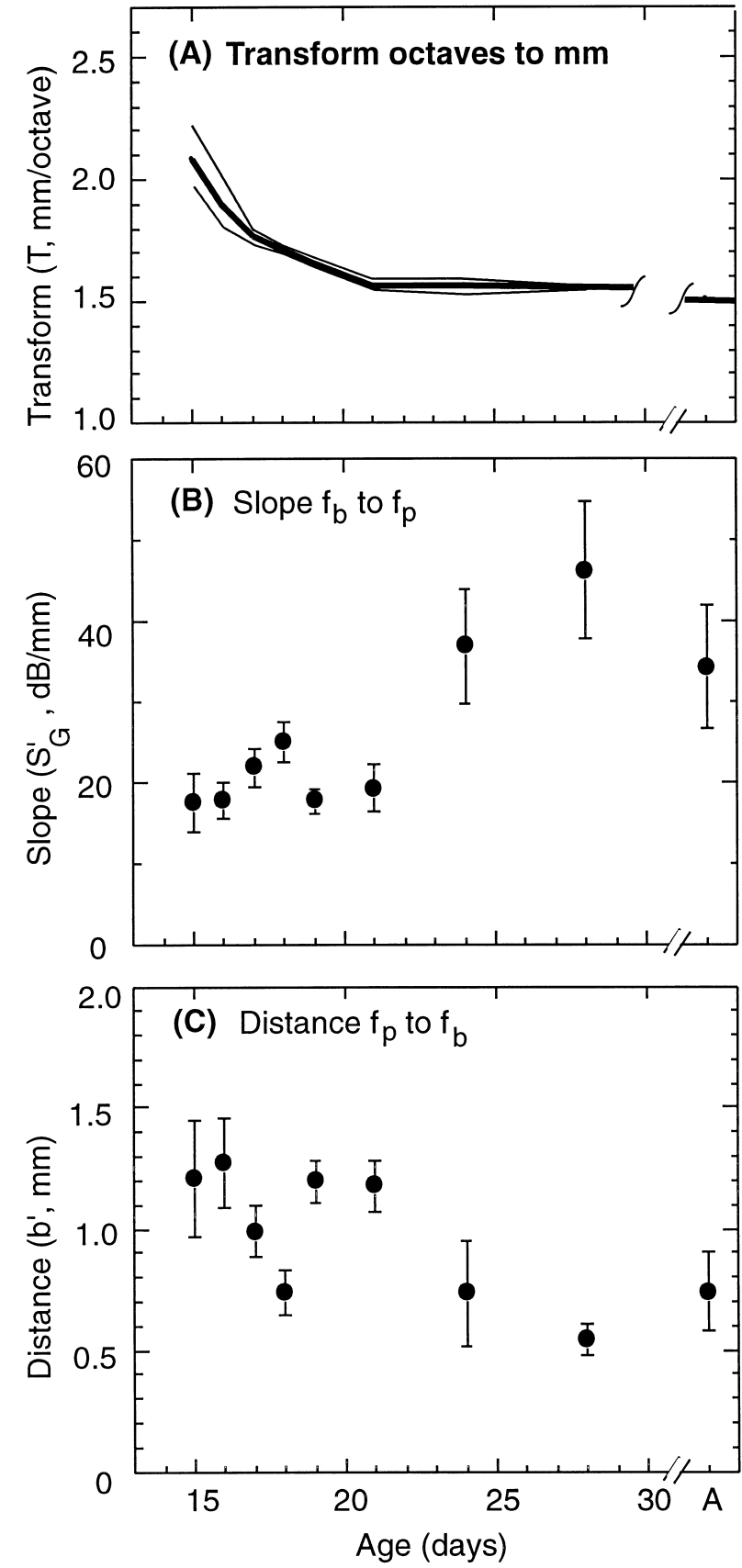

Fig. 7. Transformation of the variables in Fig. 6 from frequency to place. A: The transform ratio, $\mathrm{T}$ ( $\mathrm{mm} /$ octave) for frequencies above $2 \mathrm{kHz}$, derived from the frequencies $\mathrm{f}_{\mathrm{b}}$ in Fig. 6. The transform used is indicated by the heavy solid line, while the light lines indicate the uncertainty (S.E.M.) in the value caused by the variation in the measured $\mathrm{f}_{\mathrm{b}}$ values. $\mathrm{B}$ : The slope, $\mathrm{S}_{\mathrm{G}}{ }^{\prime}$, in $\mathrm{dB} / \mathrm{mm}$ after transformation. $\mathrm{C}$ : The corresponding distance, $\mathrm{b}^{\prime}$, transformed from octaves to $\mathrm{mm}$.

was approximately constant over the same period (Fig. 5), this implies that the slope $(\mathrm{dB} / \mathrm{mm})$ doubled during the same period. This also implies that the frequency tuning in the cochlear base improved considerably with age, at least at the extreme base. Note that while the place where this tuning improved was constant, i.e., the 


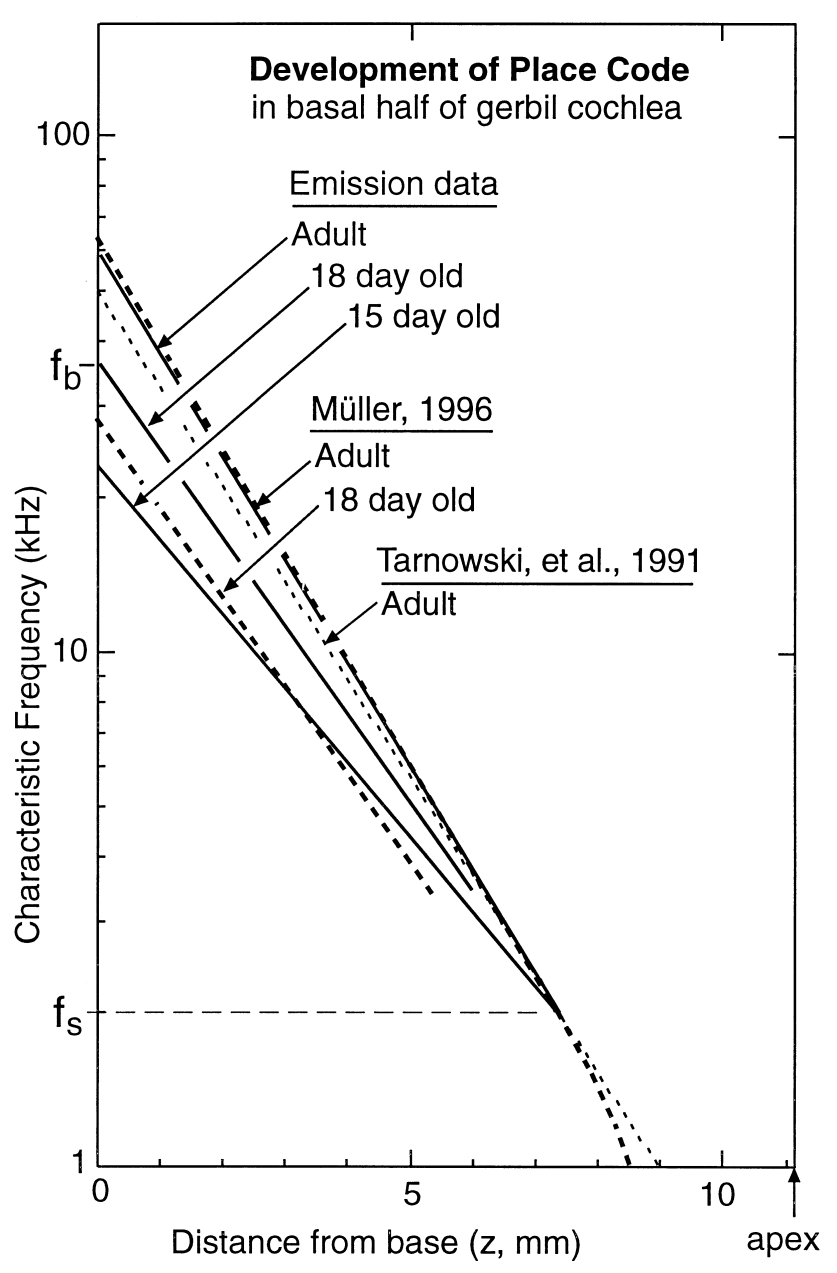

Fig. 8. Development of the frequency-place map in gerbil from emission measurements. The required assumptions are that the location of the point, denoted $f_{s}$, is assumed to remain the same during development, and is matched to Muller's (1996) adult frequencyplace map. Here, $\mathrm{f}_{\mathrm{s}}=2 \mathrm{kHz}$ (Arjmand et al., 1988). The total length of the gerbil cochlea is taken to be $11.1 \mathrm{~mm}$ for all animals from 12 days of age to adult (Müller, 1996; Harris et al., 1990). At each age, the frequency at the extreme end of the cochlea $(z=0)$, defined to be $f_{0}$, is assumed equal to the 'base cutoff frequency', $f_{b}$, at which the cochlear amplifier gain extrapolates to zero. For the example noted, $\mathrm{f}_{\mathrm{b}}=37 \mathrm{kHz}$ for the 18 day old group. The curves from Müller (1996) are the published least square fits to the traced neurons. The Tarnowski et al. (1991) relationship is placed using a total length of $11.1 \mathrm{~mm}$ (Müller, 1996). For clarity, error bars are not shown; for the emission data, these may be obtained from the $\mathrm{f}_{\mathrm{b}}$ display in Fig. 6C.

place was the extreme base, the associated frequency changed considerably with age.

The same assumptions as listed above (with Fig. 7) can be used to derive the relationship of characteristic frequency to distance along the cochlear duct, or the 'place code map', at each age (Fig. 8). That is, the base cutoff frequency, $\mathrm{f}_{\mathrm{b}}$, shown in Fig. $6 \mathrm{~A}$ is set equal to the $\mathrm{CF}$ at the base, $\mathrm{f}_{0}$. The place apical of which there is no change with age is at $\mathrm{z}=7.3 \mathrm{~mm}$, and the associated $\mathrm{CF}$ is $f_{s}=2 \mathrm{kHz}$. These derived frequency-place transforms are shown for gerbils of 15 and 18 days old, and for adults, in Fig. 8. Variances are not shown. These derived values are also compared with previous determinations of the frequency-place relationship for the adult gerbil by Müller (1996) and Tarnowski et al. (1991), and for 18 day olds by Müller (1996). The latter fit is based on only seven data points, however. In general, the agreement of the emission data with previous determinations is quite good.

\section{Discussion}

The transformation of results from a frequency to a place representation is ultimately necessary in developmental studies precisely because the frequency-place relationship changes with age (Arjmand et al., 1988; Harris and Dallos, 1984; Müller, 1996; Rubel, 1978; Rubel et al., 1984). Looked at in the frequency domain, such important measures as length of the active amplification zone for the cochlear amplifier appear to have a curvilinear response over development (Fig. 6B). However, when transformed into a representation relative to length along the cochlear duct (in $\mathrm{mm}$ ) there appears instead to be a relatively monotonic improvement (a decrease) with age (Fig. 7C).

Determination of the variation in the frequencyplace transformation ratio itself is also useful. While some aspects of the measurements are technically difficult, in principle it is a comparatively straightforward process to determine this ratio using emissions. The gain of the cochlear amplifier is determined as a function of age and frequency, with particular attention to frequencies near the upper cutoff. This allows a determination of the variation of base cutoff frequency with age. This, in turn, gives the frequency-place transformation, if a characteristic frequency in the mid or apex region can be assumed to be fixed in place with age. The frequency-place transforms derived from emission measurements have been summarized in Fig. 8, and compared with other determinations for the gerbil.

In general, the frequency-place transformation based on emission measurements agrees with existing data, and allows an extension of the relationship to younger ages (i.e., to 15 days). The emission method establishes the developmental changes in the base cutoff frequency, and relies on other measurements to establish the location of the lower frequency which does not change with age. These results indicate that emission measurements may well provide a new, relatively easy and accurate method of estimating the base cutoff frequency and the frequency-place transformation.

The frequency-place transforms illustrated in Figs. 7 and 8 may also be usefully compared with the results of the important developmental studies by Arjmand et al. (1988) and Harris and Dallos (1984). Measurements of 


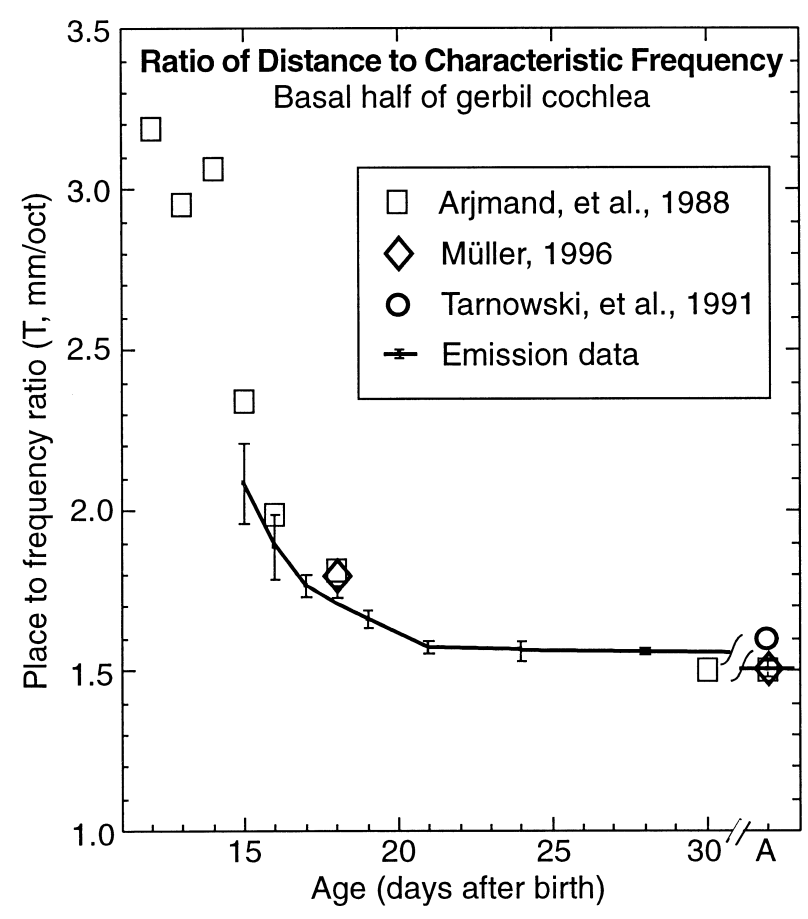

Fig. 9. The development of the slope of the frequency-place map. Heavy line and error bars: emission data, same curve as in Fig. 7. Squares: data from Arjmand et al. (1988) using the means of measured best frequency ratios between the first and second turns. The only assumption required is that their 30 day animals were essentially mature, that is, that the frequency ratio measured at this age was equivalent to the same slope as the adult data in Müller (1996), i.e., $1.5 \mathrm{~mm} /$ octave. Diamonds: slopes from Müller (1996), directly from published best fit lines for frequencies at and above $2 \mathrm{kHz}$. Circle: point for adult from Tarnowski et al. (1991) using $11.1 \mathrm{~mm}$ as a total cochlear length (Müller, 1996).

cochlear potentials were used to estimate the characteristic frequencies at two cochlear locations, the middle of the first and second turns. In the adult, the CFs found for these locations were about $16 \mathrm{kHz}$ and $2.6 \mathrm{kHz}$, respectively. The CFs at these two locations were measured in gerbils as young as 12 days after birth, with care taken that the location chosen did not change with age. Assuming only that the slope for the fit for the adult frequency-place transform in Müller (1996) is correct and that the relationship is log frequency to place, it is possible to convert the data of Arjmand et al. (1988) to find the development of the transformation ratio, $\mathrm{T}$ (mm/octave). This determination is shown in Fig. 9 for comparison with the place to frequency ratio determined from emissions (from Fig. 7). The slope of Müller's least squares fit line for the 18 day old group is also indicated. Note that, by the assumptions noted above, all the estimates for the developmental variation of $\mathrm{T}$ in Fig. 9 have the same value at maturity, equal to Müller's (1996) direct determination of $1.5 \mathrm{~mm} /$ octave.

There is quite good agreement between all three estimates. The shape of the curve as determined by emissions is very similar to that obtained by conversion of the data from Arjmand et al. (1988). The smoothness of both these curves also suggest that the changes in passive cochlear mechanics are due to an increase in the finite base cutoff frequency which occurs in a graded fashion from the base toward the apex. That is, these changes do not appear to be due to a sudden transition, but to progressive day to day changes in the physical properties of the organ of Corti.

The derived values of the ratio from the emission data appear to be slightly lower than the Arjmand et al. (1988) estimates at the youngest ages (e.g., 15 days). One possibility is that the frequencies, $f_{b}$, estimated from the gains may have been slightly high at this young age. Alternately, of course, the emission estimates may be correct and the immature cochlea may give low values when determining the $\mathrm{CF}$ by cochlear potentials. In any case, this modest discrepancy is the opposite direction from that expected if the cochlear amplifier were nonfunctional in the base region at the earliest ages tested. Therefore, the comparisons provided in Fig. 9 further support the view that the amplifier in the base of the cochlea functions more or less normally at the earliest ages tested (15 days). That is, the estimated gain appears to go sharply to zero as the frequency is increased primarily due to the passive response of the cochlea, i.e., to the lower base cutoff frequency at younger ages.

The estimate of the base cutoff frequency, as the place where this gain is extrapolated to zero, appears to be a useful addition to developmental studies of the mammalian cochlea. In particular, this approach appears to provide adequate estimates of the developmental changes in the frequency-place map (Figs. 8 and 9).

In this study, the key assumption made was that the base cutoff frequency, defined by a sharp decrease in the estimated gain of the cochlear amplifier obtained from emission measurements, was approximately equal to the characteristic frequency at the base of the cochlea, as extrapolated from direct measures using electrophysiological and neuron tracing techniques. This assumption is expected to be valid provided that the cochlear amplifier in the base region of the cochlea is functional enough at the ages tested to provide a measurable gain, so that the reason the cochlear amplifier gain goes to zero as the frequency is increased is due to the limitations on the cochlear amplifier function placed by the passive response of the cochlea. That is, because the cochlear amplifier frequency response appears to be connected to the passive frequency response of the basilar membrane, the cochlear amplifier gain must go to zero when the stimulus frequency becomes sufficiently higher than the frequency associated with the resonance of the passive basilar membrane at the extreme base of the cochlea. From these assumptions, the emission measurements led to estimates of the development of the frequency-place relationship that are in good agree- 
ment with the data from other studies (Figs. 8 and 9). This agreement then lends support to the hypothesis that the frequencies characteristic of the passive response of the base of the cochlea increase considerably with development, and that the main limitation on auditory function at high frequencies in young animals is not the absence of active mechanisms, but the limitation on active mechanisms caused by immaturity in the passive cochlear response.

The idea that the active response at the highest frequencies is limited primarily by the passive response at the base of the cochlea is supported by the detailed behavior of the emissions in this study (Figs. 3-5). This model is further supported by the observed behavioral auditory threshold responses at high frequency in adult gerbils. That is, there is a very sharp increase in threshold found as the stimulus frequency exceeds 40 $\mathrm{kHz}$ (Ryan, 1976), with a rate of about $100 \mathrm{~dB} /$ octave. Our model suggests that this sharp increase could be largely due to the decrease in cochlear length available for the active amplification to reach maximum amplitude, as the stimulus frequency approaches the base cutoff frequency, about $60 \mathrm{kHz}$ in adult gerbil. As noted above, the expected rate of threshold increase due to this effect is $G / b$, where $G$ is the normal gain of the cochlear amplifier in the base region and $b$ the normal length of the active amplification zone. For typical values for adult gerbil, $\mathrm{G}=40 \mathrm{~dB}$ (see below) and $\mathrm{b}=0.5$ octaves (Fig. 6), giving $80 \mathrm{~dB} /$ octave due to this effect alone. Base cutoff effects therefore can account for most of the observed very steep high frequency response, with the remainder probably due to middle ear dynamics. Note that the slope at very low frequencies is about $-12 \mathrm{~dB}$ /octave, most of which is probably due to middle ear dynamics.

The hypothesis that the place code shift is due to changes in passive basilar membrane mechanics is further supported by measurements of the development of the organ of Corti. The motility of outer hair cells was found to be essentially mature by 12 days of age in gerbils (He et al., 1994), consistent with the relative maturity of the cochlear amplifier in the base. In contrast, significant changes were found in the morphology of the organ of Corti in the base after 12 days of age in the same species (Schweitzer et al., 1996; Souter et al., 1997). The changes observed, a decrease in cross-sectional area and an increase in fiber thickness, are consistent with a graded increase in the passive resonant frequency of the basilar membrane in the base of the cochlea, over the period 12 days to adult in the gerbil.

The general quantitative agreement of measures found in this study with those estimated by other methods should also be considered. For the discussion above, we also need an estimate of the normal cochlear amplifier gain, $\mathrm{G}$, in adult gerbil. The estimates, $\mathrm{G}_{\mathrm{c}}$, for the cochlear amplifier gain measure at the relative max- imum frequency, $f_{p}$, were typically $25-30 \mathrm{~dB}$. These estimates are typical for frequencies throughout the cochlear base as previously reported (Mills and Rubel, 1996). As noted elsewhere, model calculations suggest a correction factor of about $10 \mathrm{~dB}$ should be added to these $G_{c}$ measures to obtain the actual cochlear amplifier gain, $G$ (Mills, 1997). Measurements using emission suppression tuning curves gave an estimate of $G=41$ $\mathrm{dB}$ for cochlear amplifier gain at $8 \mathrm{kHz}$ in an adult gerbil (Mills, 1998). These estimates actually agree quite well with tuning curves obtained from individual neurons. That is, cochlear amplifier gains can be estimated from neural tuning curves using the difference between the tip threshold and that in the 'low frequency tail'. However, care must be taken because neural tuning curves do not specify the tail frequency uniquely, and the threshold difference between tip and tail frequencies necessarily includes whatever differences there might be in passive threshold between these frequencies (Mills, 1997). With the active amplification zone of the cochlear amplifier estimated to be less than one octave (Cody, 1992), there is certainly no reason in general to consider tail frequencies much more than one octave below the tip frequency. With these considerations in mind, we estimate the cochlear amplifier gains from published tuning curves in adult gerbil (Ohlemiller and Echteler, 1990, Fig. 5) to be $\mathrm{G}=33-50 \mathrm{~dB}$, in reasonable agreement with emission estimates when suitable corrections are made, as detailed above.

\section{Conclusions}

The current results support the following consistent set of assumptions regarding cochlear function and its development at mid and high frequencies $(>2 \mathrm{kHz}$ in the gerbil).

(1) Active cochlear mechanics, i.e., the amplification of the traveling wave by the 'cochlear amplifier', are intrinsically tied to passive mechanics. The frequency which is amplified at a given location is determined by passive mechanics, i.e., by the mass and stiffness of the basilar membrane.

(2) There is a finite base cutoff frequency, above which the gain of the cochlear amplifier is zero. This frequency is set by the passive characteristics of the base of the cochlea.

(3) The base cutoff frequency increases quickly in the gerbil following the onset of hearing, rising from below $20 \mathrm{kHz}$ at 14 days after birth to above $50 \mathrm{kHz}$ at 28 days of age. This increase represents changes in the passive properties of the cochlear partition (Schweitzer et al., 1996).

(4) Setting the base cutoff frequency equal to the characteristic frequency at the base of the cochlea leads to a frequency-place map which is in good agreement 
with existing measurements, and may represent a useful method of extending more traditional measures.

(5) The effective gain of the cochlear amplifier appears to be mature, or nearly so, in the base and middle turns at or soon after the onset of hearing. That is, the cochlear amplifier appears to be capable of nearly adult levels of amplification when stimulated at age-appropriate frequencies. However, during development there is nearly a two-fold decrease in the length of the active amplification zone at the base, which is accompanied by an increase in the rate of amplification. The increase in the rate of amplification compensates for decrease in length, so that the total gain at the base remains approximately constant.

(6) Hearing at high frequencies in gerbil neonates is essentially non-existent, due to the low passive base cutoff frequency. These limitations on hearing at high frequencies in young animals are primarily due to a limited passive response, and limitation of cochlear amplifier function by the passive response.

(7) The limitation of the active process by the passive response leads to a sharp threshold increase as the stimulus frequency approaches the base cutoff frequency. The threshold increase will have a slope of about $\mathrm{G} / \mathrm{b}$, where $\mathrm{G}$ is the normal gain of the cochlear amplifier in the base and $b$ the length of the active amplification zone. This increase is expected to be observed at the uppermost range of hearing, and to extend over a distance, $b$, in octaves. The rate expected from our simple model agrees with previous determinations of the behavioral threshold in adult gerbil.

(8) While there may be small changes in place code due to modest changes in the length of the active amplification zone (Fig. 7C) the largest part of the place code shift during development appears to be driven by the changes in the base cutoff frequency. Because there is no or little place code shift in the apical part of the cochlea, the largest shifts probably occur nearest the base, while at lower frequencies there is progressively less observable shift. That is, it appears that the changes in passive cochlear mechanics due to the increase in the finite base cutoff frequency occur in a graded fashion from the base toward the apex. These changes do not appear to be due to a sudden transition, or to development of the cochlear amplifier itself, but to progressive day to day changes in the physical properties of the organ of Corti.

\section{Acknowledgments}

Thanks to S. Kujawa for comments on an earlier version of this paper. Support was provided by Research Grant DC 00395 from the National Institute on Deafness and Other Communication Disorders, National Institutes of Health.

\section{References}

Arjmand, E., Harris, D., Dallos, P., 1988. Developmental changes in frequency mapping of the gerbil cochlea: Comparison of two cochlear locations. Hear. Res. 32, 93-96.

Cody, A.R., 1992. Acoustic lesions in the mammalian cochlea: Implications for the spatial distribution of the 'active process'. Hear. Res. 62, 166-172.

Echteler, S.M., Arjmand, E., Dallos, P., 1989. Developmental alterations in the frequency map of the mammalian cochlea. Nature 341, 147-149.

Harris, D., Dallos, P., 1984. Ontogenic changes in frequency mapping in a mammalian ear. Science 225, 741-743.

Harris, D.M., Rotche, R., Freedom, T., 1990. Postnatal growth of cochlear spiral in Mongolian gerbil. Hear. Res. 50, 1-6.

He, D.Z.Z., Evans, B.N., Dallos, P., 1994. First appearance and development of electromotility in neonatal gerbil outer hair cells. Hear. Res. 78, 77-90.

Johnstone, B.M., Patuzzi, R., Yates, G.K., 1986. Basilar membrane measurements and the traveling wave. Hear. Res. 22, 147-153.

Liberman, M.C., 1982. The cochlear frequency map for the cat: labeling auditory nerve fibers of known characteristic frequency. J. Acoust. Soc. Am. 72, 1441-1449.

Lippe, W., Rubel, E.W, 1983. Development of the place principle: Tonotopic organization. Science 219, 514-516.

Manley, G.A., 1996. Ontogeny of frequency mapping in the peripheral auditory system of birds and mammals: A critical review. Audit. Neurosci. 3, 199-214.

Manley, G.A., Brix, J., Kaiser, A., 1987. Developmental stability of the tonotopic organization of the chick's basilar papilla. Science 237, 655-656.

McGuirt, J.P., Schmeidt, R.A., Schulte, B.A., 1995. Development of cochlear potentials in the neonatal gerbil. Hear. Res. 84, 52-60.

Mills, D.M., 1997. Interpretation of distortion product otoacoustic emission measurements. I. Two stimulus tones. J. Acoust. Soc. Am. 102, 413-429.

Mills, D.M., 1998. Interpretation of distortion product otoacoustic emission measurements. II. Estimating tuning characteristics using three stimulus tones. J. Acoust. Soc. Am. 103, 507-523.

Mills, D.M., Rubel, E.W, 1994. Variation of distortion product otoacoustic emissions with furosemide injection. Hear. Res. 77, 183199.

Mills, D.M., Rubel, E.W, 1996. Development of the cochlear amplifier. J. Acoust. Soc. Am. 100, 428-441.

Mills, D.M., Rubel, E.W, 1998. Development of active and passive processes in the gerbil cochlea. 135th Meeting of the Acoustical Society of America, Seattle, WA.

Mills, D.M., Norton, S.J., Rubel, E.W, 1994. Development of active and passive mechanics in the mammalian cochlea. Audit. Neurosci. 1, 77-99.

Müller, M., 1996. The cochlear place-frequency map of the adult and developing mongolian gerbil. Hear. Res. 94, 148-156.

Norton, S.J., Bargones, J.Y., Rubel, E.W, 1991. Development of otoacoustic emissions in gerbil: Evidence for micromechanical changes underlying development of the place code. Hear. Res. $51,73-92$.

Ohlemiller, K.K., Echteler, S.M., 1990. Functional correlates of characteristic frequency in single cochlear nerve fibers of the Mongolian gerbil. J. Comp. Physiol. A 167, 329-338.

Rubel, E.W, 1978. Ontogeny of structure and function in the vertebrate auditory system. In: Jacobson, M. (Ed.) Handbook of Sensory Physiology, Springer-Verlag, New York, pp. 135-237.

Rubel, E.W, Ryals, B.M., 1983. Development of the place principle: Acoustic trauma. Science 219, 512-514.

Rubel, E.W, Lippe, W.R., Ryals, B.M., 1984. Development of the place principle. Annals Otol. Rhinol. Laryngol. 6, 609-615. 
Rübsamen, R., 1992. Postnatal development of central auditory frequency maps. J. Comp. Physiol. 170, 129-143.

Ryan, A.F., 1976. Hearing sensitivity of the mongolian gerbil Meriones unguiculatis. J. Acoust. Soc. Am. 59, 1222-1226.

Ryan, A.F., Woolf, N.K., 1992. Development of the lower auditory system in the gerbil. In: Romand, R. (Ed.), Development of Auditory and Vestibular Systems II, Elsevier, Amsterdam, pp. 243271.

Sanes, D.H., Rubel, E.W, 1988. Development of stimulus coding in the auditory system. In: Jahn, A.F., Santos-Sacchi, J.R. (Eds.), Physiology of the Ear, Raven Press, New York, pp. 431-455.

Sanes, D.H., Merickel, M., Rubel, E.W, 1989. Evidence for an alteration of the tonotopic map in the gerbil cochlea during development. J. Comp. Neurol. 279, 436-444.

Schmiedt, R.A., Adams, J.C., 1981. Stimulated acoustic emissions in the ear canal of the gerbil. Hear. Res. 5, 295-305.

Schweitzer, L., Lutz, C., Hobbs, M., Weaver, S., 1996. Anatomical correlates of the passive properties underlying the developmental shift in the frequency map of the mammalian cochlea. Hear. Res. 97, 84-94.

Smith, D.I., Kraus, N., 1987. Postnatal development of the auditory brainstem response (ABR) in the unanesthetized gerbil. Hear. Res. 27, 157-164.

Souter, M., Nevell, G., Forge, A., 1997. Postnatal maturation of the organ of Corti in gerbils: Morphology and physiological responses. J. Comp. Neurol. 386, 635-651.
Tarnowski, B.I., Schmiedt, R.A., Hellstrom, L.I., Lee, F.S., Adams, J.C., 1991. Age-related changes in cochleas of mongolian gerbils. Hear. Res. 54, 123-134.

von Békésy, G., 1960. Experiments in Hearing, trans. E.G. Wever, McGraw-Hill, New York.

Walsh, E.J., Romand, R., 1992. Functional development of the cochlea and the cochlear nerve. In: Romand, R. (Ed.), Development of Auditory and Vestibular Systems II, Elsevier, Amsterdam, pp. $161-219$.

Woolf, N.K., Ryan, A.F., 1984. The development of auditory function in the cochlea of the mongolian gerbil. Hear. Res. 13, 277283.

Woolf, N.K., Ryan, A.F., 1985. Ontogeny of neural discharge patterns in the ventral cochlear nucleus of the Mongolian gerbil. Dev. Brain Res. 17, 131-147.

Woolf, N.K., Ryan, A.F., 1988. Contributions of the middle ear to the development of function in the cochlea. Hear. Res. 35, 131142.

Woolf, N.K., Ryan, A.F., Harris, J.P., 1986. Development of mammalian endocochlear potential: normal ontogeny and effects of anoxia. Am. J. Physiol. 250, R493-R498.

Yancey, C., Dallos, P., 1985. Ontogenic changes in cochlear characteristic frequency at a basal turn location as reflected in the summating potential. Hear. Res. 18, 189-195.

Zweig, G., 1991. Finding the impedance of the organ of Corti. J. Acoust. Soc. Am. 89, 1229-1254. 